\title{
Errata
}

Errata to 'An Alternative Construction of Conway's Ordered Field No,' Philip Ehrlich, Algebra Universalis 25 (1988) 7-16.

Due to an oversight, the following paragraph was deleted from the beginning of the proof of Lemma 2. Please insert:

First note that $S$ is an $\aleph_{\alpha}$-universally extending ordered field (resp. ordered abelian group) iff $S$ is an $\aleph_{\alpha}$-universally extending real-closed ordered field (resp. divisible ordered abelian group). [Necessity is assured by the familiar embedding theorems of Artin-Schreier (resp. Baer-Clifford) (cf. [30], p. 148), and sufficiency may be established by using the relevant portions of the respective embedding theorems to identify a chain of real-closed subfields (resp. divisible subgroups) of $S$ whose union equals $S$.] Consequently, the proofs of equivalence and uniqueness may proceed in accordance with the prescription: all relevant fields (resp. groups) are either known to be or are assumed to be real-closed (resp. divisible).

\section{Typographical errors}

p. 10 On line -7 , for 'of $S$ ' read 'of $A$ in $S$ '; on line -4 , for 'linearally' read 'linearly'; and on the last line, for 'image' read 'images'.

p. 11 On line 9 , for ' $A=\left(g\left((X \cup Y)_{S} \cup\{e\}\right)\right)_{E}$ ' read ' $A=\left(g\left((X \cup Y)_{S}\right) \cup\right.$ $\{e\})_{E}$ '.

p. 12 On line 13 , for ' $2 \kappa_{\text {On }}>\aleph_{\text {On }}$ ' read ' $2 \aleph^{\aleph_{\text {on }}}>\kappa_{\text {On }}$ '. 